\title{
CORRIGENDUM
}

\section{Diminished upregulation of visceral adipose heme oxygenase-1 correlates with waist to hip ratio and insulin resistance}

S Shakeri-Manesch, M Zeyda, J Huber, B Ludvik, G Prager and TM Stulnig

International Journal of Obesity (2010) 34, 1102; doi:10.1038/ijo.2009.272

Correction to: International Journal of Obesity (2009) 33, 1257-1264; doi:10.1038/ijo.2009.160

An error has occurred within the Methods section of this paper. The first sentence of the Paragraph entitled 'Patients' should read as follows:

'Paired samples of visceral (omental) and subcutaneous adipose tissue were obtained from 40 Caucasian men $(n=8)$ and women $(n=32)$.'
Hence, there are 16 obese women in the study population as correctly stated in the legend to Figure 3a. This typographic error does not affect any results or conclusions of the study. The authors apologize for the error and the potential confusion it could have caused. 